\title{
Zeeman spectroscopy of CaH molecules in a magnetic trap
}

\section{Citation}

Friedrich, Bretislav, Jonathan D. Weinstein, Robert deCarvalho, and John M. Doyle. 1999. "Zeeman Spectroscopy of CaH Molecules in a Magnetic Trap." J. Chem. Phys. 110 (5): 2376. doi:10.1063/1.477942.

\section{Published Version}

doi:10.1063/1.477942

\section{Permanent link}

http://nrs.harvard.edu/urn-3:HUL.InstRepos:28421227

\section{Terms of Use}

This article was downloaded from Harvard University's DASH repository, and is made available under the terms and conditions applicable to Other Posted Material, as set forth at http:// nrs.harvard.edu/urn-3:HUL.InstRepos:dash.current.terms-of-use\#LAA

\section{Share Your Story}

The Harvard community has made this article openly available.

Please share how this access benefits you. Submit a story.

Accessibility 


\title{
Zeeman spectroscopy of $\mathrm{CaH}$ molecules in a magnetic trap
}

\author{
Bretislav Friedrich, ${ }^{\text {a) }}$ Jonathan D. Weinstein, Robert deCarvalho, and John M. Doyle \\ Department of Physics, Harvard University, Cambridge, Massachusetts 02138
}

(Received 28 September 1998; accepted 27 October 1998)

In a recent experiment [Weinstein et al., Nature 395, 148 (1998)] we magnetically trapped $10^{8}$ ground-state calcium monohydride molecules, $\mathrm{CaH}\left(X^{2} \Sigma, v^{\prime \prime}=0, J^{\prime \prime}=0\right)$. The molecules were prepared by laser ablation of a solid sample of $\mathrm{CaH}_{2}$ and loaded via thermalization with a cold $(<1 \mathrm{~K}){ }^{3} \mathrm{He}$ buffer gas. The magnetic trap was formed by superconducting coils arranged in the anti-Helmholtz configuration. The detection was done by laser fluorescence spectroscopy excited at $635 \mathrm{~nm}$ (in the $B^{2} \Sigma, v^{\prime}=0-X^{2} \Sigma, v^{\prime \prime}=0$ band) and detected at $692 \mathrm{~nm}$ (within the $B, v^{\prime}=0$ $-X, v^{\prime \prime}=1$ band). Both a photomultiplier tube and a CCD camera were used. Due to the thermalization of molecular rotation, only a transition from the lowest rotational state could be detected at zero field, $N^{\prime}=1, J^{\prime}=3 / 2 \leftarrow N^{\prime \prime}=0, J^{\prime \prime}=1 / 2$. In the magnetic field this rotational transition splits into two features, one shifted towards lower and one towards higher frequencies. The measured shifts are linear in field strength and indicate a small difference $\left(0.02 \mu_{B}\right)$ in the magnetic moments between the ground and excited states. Here we present a theoretical analysis of the observed magnetic shifts. These are identified as arising from a rotational perturbation of the $B^{2} \Sigma, v^{\prime}=0$ state by a close-lying $A^{2} \Pi, v^{\prime}=1$ state that lends the $B$ state some of its $A$ character. We find that the Hamiltonian can be well approximated by a $3 \times 3$ matrix built out of elements that connect states from within the $\Sigma$-doublet and the ${ }^{2} \Pi_{3 / 2}$ manifolds. The interaction parameter describing the $\Sigma-\Pi$ coupling in the Zeeman Hamiltonian is determined from the observed shifts and the field-free molecular parameters of $\mathrm{CaH}$ given by Berg and Klyning [Phys. Scr. 10, 331 (1974)] and by Martin [J. Mol. Spectrosc 108, 66 (1984)]. (C) 1999 American Institute of Physics. [S0021-9606(99)00805-3]

\section{INTRODUCTION}

The advent of molecular trapping presents a new challenge to the spectroscopy of molecules in external magnetic $^{1,2}$ and electric ${ }^{3,4}$ fields. In particular, the wide applicability of buffer-gas loaded magnetic traps to many paramagnetic molecules (and atoms) $)^{1,5}$ stimulates a renewed interest in high-resolution Zeeman spectroscopy. The trap spectroscopy is not only vital to the understanding of the processes that take place within the confines of the trapping field but, equally importantly, benefits the elucidation of the structure of the molecules themselves.

In a recent experiment ${ }^{2}$ we magnetically trapped over $10^{8}$ ground-state calcium monohydride molecules, $\mathrm{CaH}\left(X^{2} \Sigma, v^{\prime \prime}=0\right)$, at a temperature of about $400 \mathrm{mK}$ and performed high-resolution Zeeman spectroscopy on the trapped ensemble in the $B-X$ band. ${ }^{6,7}$

The principle of buffer-gas loading and a detailed description of our apparatus are given in our previous papers. ${ }^{5,89}$ Briefly, our magnetic trap is a linear quadrupole field formed by two superconducting coils arranged in an anti-Helmholtz configuration, see Fig. 1. In the center between the two coils is a zero field point from which the magnitude of the field increases linearly over the trapping region in any direction. This configuration confines molecules that are in the low field seeking states (i.e., states

\footnotetext{
a) Also at Department of Chemistry and Chemical Biology, Harvard University, Cambridge, MA 02138.
}

whose energy increases with increasing field strength; as a result, they seek regions of minimum field strength where their energy is lowest). Because the magnetic dipole moment of ground-state $\mathrm{CaH}$ is $1 \mu_{B}$ (Bohr magneton) and the maximum attainable magnetic field strength at the trap edge is $\mathcal{H}_{\text {edge }} \approx 3 \mathrm{~T}$, the maximum depth of the magnetic trap is $2 \mathrm{~K}$. $\mathrm{CaH}$ molecules are produced and trapped within a copper cell located in the bore of the magnet. The cell is filled with ${ }^{3} \mathrm{He}$ buffer gas and its temperature is maintained by thermal contact with a dilution refrigerator. Typically, the ${ }^{3} \mathrm{He}$ number density is about $10^{16} \mathrm{~cm}^{-3}$. The bottom of the cell is outfitted with a fused silica window to enable optical access. The $\mathrm{CaH}$ molecules are created by ablating a solid sample of $\mathrm{CaH}_{2}$ placed within the cell at the edge of the trapping region with a $10 \mathrm{~mJ}, 7 \mathrm{~ns}$ pulse of a frequency-doubled YAG laser. The initially hot $\mathrm{CaH}$ molecules diffuse through the helium gas and quickly thermalize with it via elastic collisions.

The $\mathrm{CaH}$ molecules are detected by laser fluorescence spectroscopy. The fluorescence is excited at $635 \mathrm{~nm}$ in the $B^{2} \Sigma, v^{\prime}=0-X^{2} \Sigma, v^{\prime \prime}=0$ band and detected mainly within the $B, v^{\prime}=0-X, v^{\prime \prime}=1$ band at $692 \mathrm{~nm}$ (the corresponding Franck-Condon factor was calculated to be $0.028,{ }^{10}$ ). A set of color-glass and band-pass interference filters placed in front of the detector (either a photomultiplier tube or a CCD camera) serves to block the scattered probe radiation (along with the $B, v^{\prime}=0-X, v^{\prime \prime}=0$ fluorescence).

Typically, under field free conditions, only a single rotational transition, $N^{\prime}=1, J^{\prime}=3 / 2 \leftarrow N^{\prime \prime}=0, J^{\prime \prime}=1 / 2$, can be detected. This is consistent with a fast rotational relaxation 


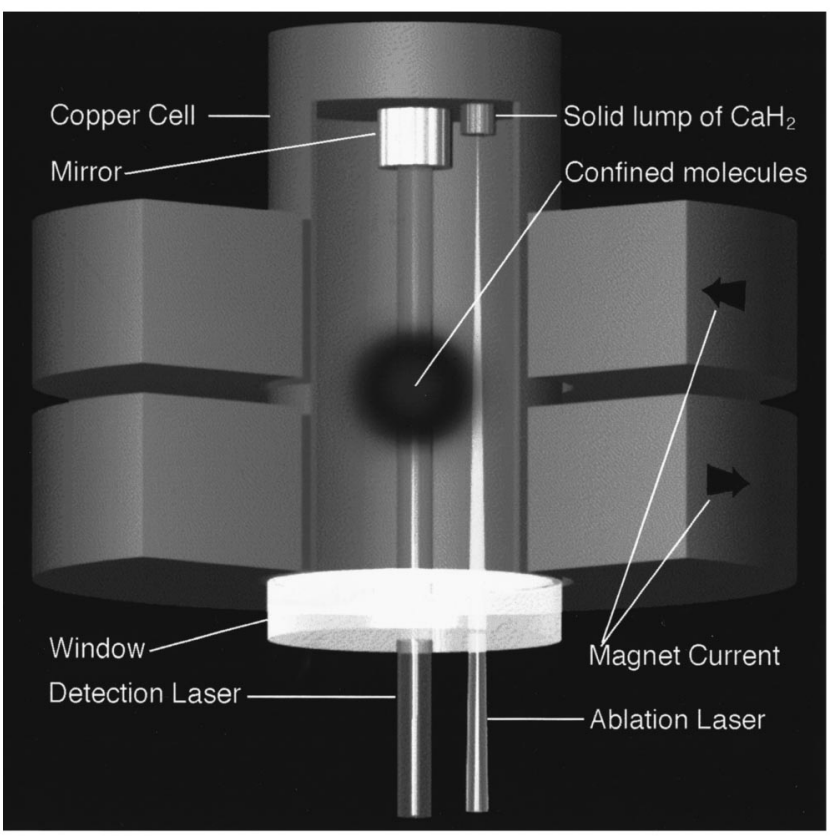

FIG. 1. Schematic of apparatus. The copper cell is anchored to the mixing chamber of a dilution refrigerator. The magnet is immersed in liquid helium. Vacuum isolates the relatively warm $(4 \mathrm{~K})$ magnet from the cold $(300 \mathrm{mK})$ cell. The superconducting magnet coils are arranged in the anti-Helmholtz configuration (currents travel in opposite directions) and form a magnetic trap up to $3 \mathrm{~T}$ deep. Detection and ablation lasers enter through three borosilicate windows (not shown) at $300 \mathrm{~K}, 77 \mathrm{~K}$, and $4 \mathrm{~K}$ before passing through the fused silica window at the bottom of the cell. Fluorescence from the molecules (induced by the probe laser) reflected off a mirror mounted on the top of the cell is collected outside the $300 \mathrm{~K}$ window by a photomultiplier tube or a CCD camera.

of the molecules that renders the population of higher rotational states negligible: the next rotational state lies $8.5 \mathrm{~cm}^{-1}$ above the $N^{\prime \prime}=0, J^{\prime \prime}=1 / 2$ ground state and the intensity of the corresponding transition is well below $0.1 \%$ of the $N^{\prime}$ $=1, J^{\prime}=3 / 2 \leftarrow N^{\prime \prime}=0, J^{\prime \prime}=1 / 2$ transition. This yields an upper limit on the rotational temperature of $1.5 \mathrm{~K}$. Using absorption spectroscopy to calibrate the fluorescence detection, we found that up to $10^{10} \mathrm{CaH}$ molecules could, under certain conditions, be formed by a single ablation pulse. We were also able to detect the formation of $X^{2} \Sigma, v^{\prime \prime}=1$ molecules which were about 10-100 fewer; their scarcity precluded them from being detected in the trap. However, we determined the upper limit of the $v^{\prime \prime}=1 \rightarrow v^{\prime}=0$ relaxation cross section (in collisions with the ${ }^{3} \mathrm{He}$ buffer gas) to be $10^{-18} \mathrm{~cm}^{2}$ which suggests that it should be possible to load them into the trap.

As the magnetic field is turned up, the field-free rotational transition is observed to split into two features shifted towards lower and higher frequencies. Figures $2 \mathrm{a}$ and $2 \mathrm{~b}$ display, respectively, spectra with negative and positive frequency shifts taken at different values of $\mathcal{H}_{\text {edge }}$. All panels correspond to delay times of up to $25 \mathrm{~ms}$ (with respect to the ablation pulse) when molecules in both the low- and highfield seeking states are spread almost evenly over the cell and their distributions are nearly symmetric, peaked towards high field. This shape reflects the available phase space which increases towards the edge of the trap due to the spherical geometry of the field and is further enhanced at the edge due to the saddle point. As described in our previous paper, ${ }^{2}$ at
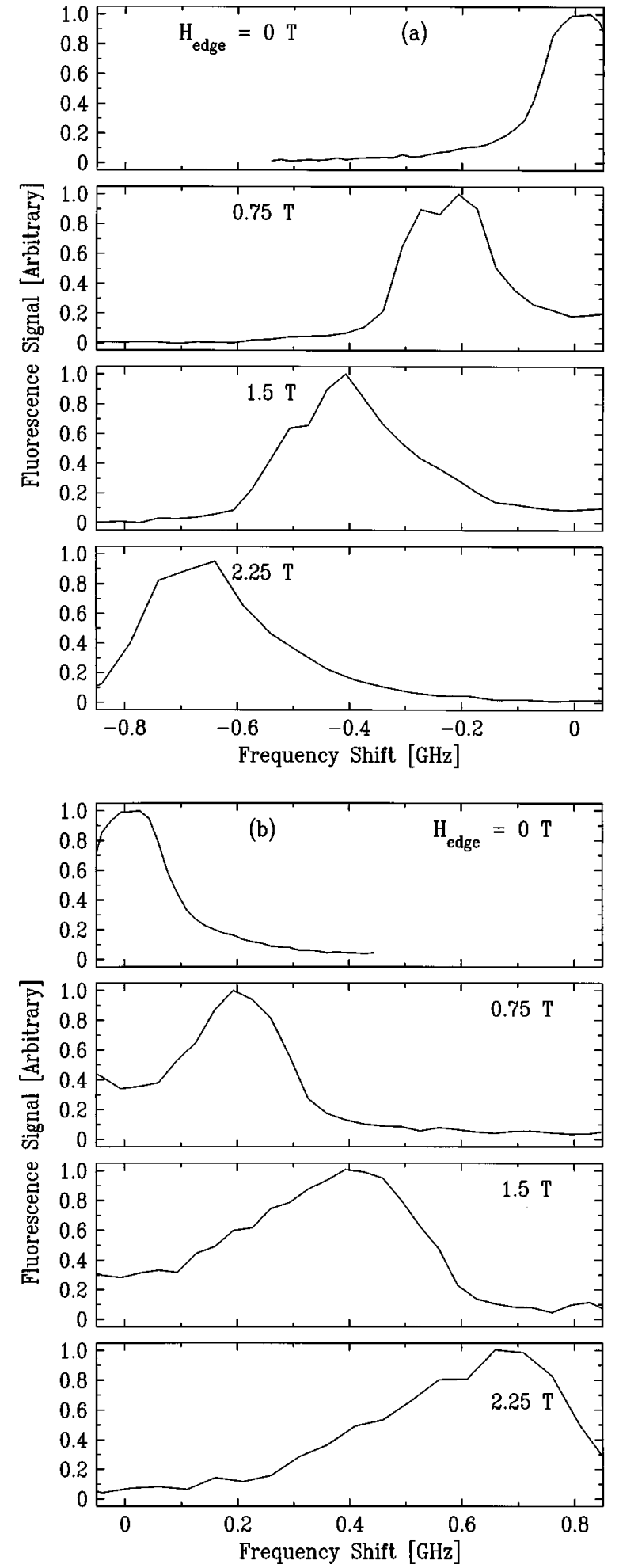

FIG. 2. Spectra taken at different values of the magnetic field at the edge of the trap, $\mathcal{H}_{\text {edge }}$. All panels correspond to delay times of up to $25 \mathrm{~ms}$ (with respect to the ablation pulse). The frequency scale is relative to the frequency $\tilde{\nu}_{0}=15762.96 \mathrm{~cm}^{-1}$ of the $R_{1}(1 / 2)$ field-free transition (Ref. 11). Part (a) shows spectra with a negative frequency shift corresponding to high-field seeking states; part (b) shows spectra with a positive frequency shift corresponding to low-field seeking states. All spectra are normalized to the peak height.

later times, the high-field seekers move towards the edge of the trap, hit the wall of the cell, stick to it and are lost; the low-field seekers, on the other hand, move towards the center of the trap and by $300 \mathrm{~ms}$ their distribution is that of a 


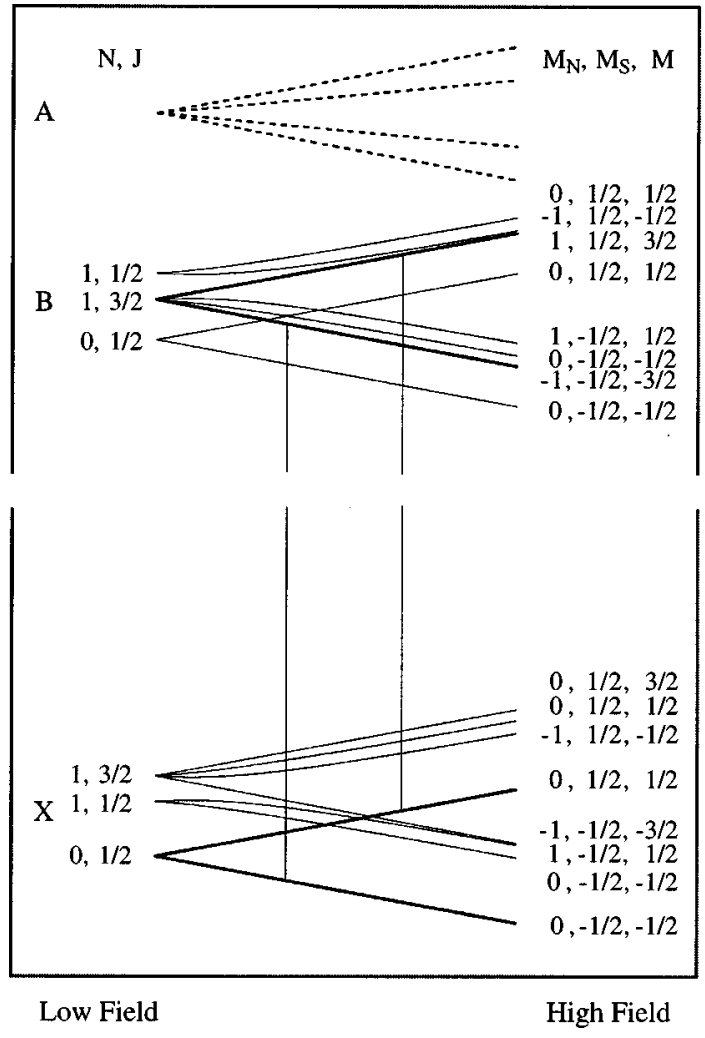

FIG. 3. A correlation diagram between the low- and high-field limits for states from within the $X, v^{\prime \prime}=0$ and $B, v^{\prime}=0$ manifolds; also shown are the perturbing states from within the $A, v^{\prime}=1$ manifold (dashed). The states from within the $X$ and $B$ manifolds are labeled by the Hund's case (b) angular momentum quantum numbers: $N$ (rotation) and $J$ (total, excluding nuclear spin) in the low-field limit and their projections on the direction of the field (the $\mathbf{Z}$ axis), $M_{N}$ and $M$, in the high-field limit. The slopes of the eigenenergies in the high-field limit are determined by the projection, $M_{S}$, of the spin angular momentum on $\mathbf{Z}$. The states involved in the spectroscopic transitions detected in our experiment are shown by bold lines; the transitions are indicated by vertical lines.

trapped ensemble at $T=400 \mathrm{mK}$, close to the initial temperature of the buffer gas of $300 \mathrm{mK}$.

Here we give a theoretical analysis that assigns the feature that shifts towards higher frequencies to the transition from the $N^{\prime \prime}=0, J^{\prime \prime}=1 / 2, M^{\prime \prime}=1 / 2$ low-field seeking state to the $N^{\prime}=1, J^{\prime}=3 / 2, M^{\prime}=3 / 2$ state, and the feature that shifts towards lower frequencies to the transition from the $N^{\prime \prime}$ $=0, J^{\prime \prime}=1 / 2, M^{\prime \prime}=-1 / 2$ high-field seeking state to the $N^{\prime}$ $=1, J^{\prime}=3 / 2, M^{\prime}=-3 / 2$ state. We find that the slopes of the $N^{\prime}=1, J^{\prime}=3 / 2, M^{\prime}= \pm 3 / 2$ states differ from the slopes of the $N^{\prime \prime}=0, J^{\prime \prime}=1 / 2, M^{\prime \prime}= \pm 1 / 2$ due to a perturbation of the $B^{2} \Sigma, v^{\prime}=0$ by a close-lying $A^{2} \Pi, v^{\prime}=1$ that lends the $B$ state some of its $A$ character.

Figure 3 shows a correlation diagram between the lowand high-field limits for states from within the $X, v^{\prime \prime}=0$ and $B, v^{\prime \prime}=0$ manifolds; also shown are the perturbing states from within the $A, v^{\prime}=1$ manifold (dashed). The states from within the $X$ and $B$ manifolds are labeled by the Hund's case (b) angular momentum quantum numbers: $N$ (rotation) and $J$ (total, excluding nuclear spin) in the low-field limit and their projections on the direction of the field (the $Z$ axis), $M_{N}$ and $M$, in the high-field limit. The slopes of the eigenenergies in the high-field limit are determined by the projection, $M_{S}$, of the spin angular momentum on $Z$ (the Paschen-Back uncoupling). The states involved in the spectroscopic transitions detected in our experiment are shown by bold lines. Note the different order of the eigenenergies in the $X$ and $B$ state manifolds in either limit—due to the opposite signs of the spin-rotation coupling constant. ${ }^{6,7}$ While the field dependence of the states correlating with $N^{\prime \prime}=0, J^{\prime \prime}=1 / 2$ from within the $X$-state manifold is trivial, given by $\pm g_{S} \mathcal{H} / 2$ (with $g_{S}$ the electron spin gyromagnetic ratio) for $M^{\prime \prime}$ $= \pm 1 / 2$, the Zeeman curves of the $N^{\prime}=1, J^{\prime}=3 / 2, M^{\prime}$ $= \pm 3 / 2, \pm 1 / 2$ states from within the $B^{2} \Sigma^{+}, v^{\prime}=0$ state manifold are affected by a perturbation from the $A, v^{\prime}=1$ state. Their computation is the subject of the following section.

\section{THEORY: THE HAMILTONIAN AND ITS MATRIX ELEMENTS IN A BASIS SET INTERMEDIATE BETWEEN HUND'S CASES, (a) AND (b)}

\section{A. Field-free rotational perturbations}

In the absence of an external field, the Hamiltonian of a linear molecule is given by the sum of the vibronic, rotational, spin-spin, and spin-orbit Hamiltonians (see Appendix):

$$
H_{0}=H_{e v}+H_{r}+H_{s s}+H_{s o} .
$$

In what follows we will consider the interaction of a ${ }^{2} \Sigma, v_{\Sigma}$ with a ${ }^{2} \Pi, v_{\Pi}$ vibronic state. For this case, Hamiltonian (1) simplifies since $H_{s s}=0$, as in any doublet state. Hence, the ${ }^{2} \Sigma, v_{\Sigma} \sim{ }^{2} \Pi, v_{\Pi}$ perturbation (described by the off-diagonal matrix elements of $H_{0}$ ) is due to the vibronic overlap and the $L$ - and $S$-uncoupling and spin-orbit interactions.

In order for two rotational states to interact, their total angular momenta (excluding nuclear spin), $\mathbf{J}$, and their parities, $\mathbf{P}$, must be the same and the overlap of the vibrational eigenfunctions of the two states must be nonzero. ${ }^{12}$ Therefore, to construct a suitable representation of the field-free Hamiltonian, we need a basis set of states with definite parity that correspond to the three electronic states in question: a ${ }^{2} \Sigma$ state $(|\Omega|=1 / 2)$ and two ${ }^{2} \Pi$ states (one with $|\Omega|=1 / 2$ and one with $|\Omega|=3 / 2$ ). Such a basis set, describing an intermediate coupling between Hund's cases (a) and (b), has been introduced by Radford and Broida: ${ }^{13}$

$$
\begin{aligned}
& \left|1, \frac{c}{d}, J\right\rangle=(2)^{-1 / 2}\left({ }^{2} \Sigma_{1 / 2} \pm{ }^{2} \Sigma_{-1 / 2}\right), \\
& \left|2, \frac{c}{d}, J\right\rangle=a\left({ }^{2} \Pi_{3 / 2} \pm{ }^{2} \Pi_{-3 / 2}\right)-b\left({ }^{2} \Pi_{1 / 2} \pm{ }^{2} \Pi_{-1 / 2}\right), \\
& \left|3, \frac{c}{d}, J\right\rangle=b\left({ }^{2} \Pi_{3 / 2} \pm{ }^{2} \Pi_{-3 / 2}\right)+a\left({ }^{2} \Pi_{1 / 2} \pm{ }^{2} \Pi_{-1 / 2}\right),
\end{aligned}
$$

where

$$
\begin{aligned}
& a \equiv\left[\frac{X+(2-\lambda)}{4 X}\right]^{1 / 2}, \\
& b \equiv\left[\frac{X-(2-\lambda)}{4 X}\right]^{1 / 2},
\end{aligned}
$$




$$
\begin{aligned}
\lambda & \equiv \frac{A}{B_{\Pi}}, \\
X & \equiv\left[4\left(J+\frac{1}{2}\right)^{2}+\lambda(\lambda-4)\right]^{1 / 2},
\end{aligned}
$$

and the upper and lower sign pertains respectively to the $c$ and $d$ symmetry. Note that $|1,(c / d), J\rangle \rightarrow{ }^{2} \Sigma_{|1 / 2|}$ and for $\lambda$ $\rightarrow-\infty,|2,(c / d), J\rangle \rightarrow{ }^{2} \Pi_{|3 / 2|}$ and $|3,(c / d), J\rangle \rightarrow{ }^{2} \Pi_{|1 / 2|}$; for $\lambda \rightarrow \infty,|2,(c / d), J\rangle \rightarrow{ }^{2} \Pi_{|1 / 2|}$ and $|3,(c / d), J\rangle \rightarrow{ }^{2} \Pi_{|3 / 2|}$.

Using the matrix elements (A1)-(A8) of the Appendix, we obtain for the matrix elements of $H_{0}$ :

$$
\begin{aligned}
& \left\langle 1, \frac{c}{d}, J\left|H_{0}\right| 1, \frac{c}{d}, J\right\rangle=\alpha \pm \delta, \\
& \left\langle 2, \frac{c}{d}, J\left|H_{0}\right| 2, \frac{c}{d}, J\right\rangle=2\left(a^{2} \gamma-2 a b \varepsilon+b^{2} \beta\right), \\
& \left\langle 3, \frac{c}{d}, J\left|H_{0}\right| 3, \frac{c}{d}, J\right\rangle=2\left(b^{2} \gamma+2 a b \varepsilon+a^{2} \beta\right), \\
& \left\langle 1, \frac{c}{d}, J\left|H_{0}\right| 2, \frac{c}{d}, J\right\rangle=2^{1 / 2}[a \eta \mp b(\xi \pm \theta)], \\
& \left\langle 1, \frac{c}{d}, J\left|H_{0}\right| 3, \frac{c}{d}, J\right\rangle=2^{1 / 2}[b \eta+a(\theta \pm \xi)], \\
& \left\langle 2, \frac{c}{d}, J\left|H_{0}\right| 3, \frac{c}{d}, J\right\rangle=2\left[a^{2} \varepsilon+a b(\gamma-\beta)-b^{2} \varepsilon\right] .
\end{aligned}
$$

In this formulation, the dependence of the eigenstates of $H_{0}$ on the electronic motion and the nuclear vibration is contained in the parameters $\xi=\xi(Q), \eta=\eta(Q)$, and $\theta$ $=\theta(Q, P)$ that depend on the perturbation parameters $Q$ and $P$, see Eqs. (A9) and (A10) of the Appendix.

\section{B. Rotational perturbation in the presence of an external magnetic field}

The Hamiltonian of a molecule subject to a uniform magnetic field $\mathcal{H}$ is given by

$$
H=H_{0}+H_{Z},
$$

where the Zeeman Hamiltonian is

$$
H_{Z}=-\mu_{Z} \mathcal{H}
$$

see Eq. (A11) of the Appendix. The nonvanishing matrix elements of $H_{Z}$ in the Radford-Broida basis set are:

$$
\begin{aligned}
& \left\langle 1, c, J, M\left|H_{Z}\right| 1, c, J, M\right\rangle=-\frac{M}{J+1} \mu_{B} \mathcal{H}, \\
& \left\langle 1, d, J, M\left|H_{Z}\right| 1, d, J, M\right\rangle=\frac{M}{J} \mu_{B} \mathcal{H}, \\
& \left\langle 2, \frac{c}{d}, J, M\left|H_{Z}\right| 2, \frac{c}{d}, J, M\right\rangle \\
& \quad=\left[\left(1+\frac{g_{S}}{2}\right) a^{2} \frac{3 M}{J(J+1)}+2 g_{S} a b t M\right] \mu_{B} \mathcal{H},
\end{aligned}
$$

$$
\begin{aligned}
& \left\langle 3, \frac{c}{d}, J, M\left|H_{Z}\right| 3, \frac{c}{d}, J, M\right\rangle \\
& \quad=\left[\left(1+\frac{g_{S}}{2}\right) b^{2} \frac{3 M}{J(J+1)}-2 g_{S} a b t M\right] \mu_{B} \mathcal{H},
\end{aligned}
$$

$$
\begin{aligned}
& \left\langle 1, \frac{c}{d}, J, M\left|H_{Z}\right| 2, \frac{c}{d}, J, M\right\rangle \\
& \quad=-2^{-1 / 2} L_{\Pi, \Sigma} M(a t \mp b u) \mu_{B} \mathcal{H},
\end{aligned}
$$

$$
\begin{aligned}
& \left\langle 1, \frac{c}{d}, J, M\left|H_{Z}\right| 3, \frac{c}{d}, J, M\right\rangle \\
& \quad=-2^{-1 / 2} L_{\Pi, \Sigma} M(b t \pm a u) \mu_{B} \mathcal{H}
\end{aligned}
$$

$$
\begin{aligned}
& \left\langle 2, \frac{c}{d}, J, M\left|H_{Z}\right| 3, \frac{c}{d}, J, M\right\rangle \\
& \quad=\left(b^{2}-a^{2}\right) g_{S} t \mu_{B} \mathcal{H}+a b\left(1+\frac{g_{S}}{2}\right) \frac{3 M}{J(J+1)} \mu_{B} \mathcal{H},
\end{aligned}
$$

with

$t \equiv \frac{\left[J(J+1)-\left(\frac{3}{4}\right)\right]^{1 / 2}}{J(J+1)}$,

$u \equiv \frac{\left[J+\left(\frac{1}{2}\right)\right]}{J(J+1)}$.

Note that the off-diagonal Zeeman matrix elements (21) and (23) modify the field-free perturbations given by the matrix elements (12) and (14). Also, note that states with the same $c / d$ symmetry have the same parity but not vice versa.

Apart from the matrix elements that connect states with same $J$ and $M$ there are non-vanishing matrix elements between states with same $M$ and parity but with $J$ 's that differ by $\Delta J= \pm 1$. These matrix elements have always to be included for the other member of the $\Sigma$-doublet since members of a $\Sigma$-doublet are quasi-degenerate and have the same parity. On the other hand, in the case of a large spin-orbit splitting between the ${ }^{2} \Pi_{|1 / 2|}$ and ${ }^{2} \Pi_{|3 / 2|}$ states (or, more accurately, large $\lambda$ ), there can only be a significant interaction between the ${ }^{2} \Sigma_{|1 / 2|}$ state and just one of the ${ }^{2} \Pi$ states (the one that exhibits an accidental quasi-degeneracy with the ${ }^{2} \Sigma_{|1 / 2|}$ state). Therefore, the Hamiltonian can be well approximated by a $3 \times 3$ matrix built out of elements that connect the $\Sigma$-doublet with either the ${ }^{2} \Pi_{|1 / 2|}$ or the ${ }^{2} \Pi_{|3 / 2|}$ state. Note that the other member of the ${ }^{2} \Pi_{|1 / 2|}$ or ${ }^{2} \Pi_{|3 / 2|} \Lambda$ doublet does not interact with the three states because of its different parity.

Thus, in the case of a quasi-degeneracy, for given vibronic states and a given $J$, between the $|1, c, J, M\rangle$ and $|2, c, J, M\rangle$ states of positive parity, the $3 \times 3$ Hamiltonian matrix will also contain matrix elements that connect these two states with the $|1, d, J+1, M\rangle$ state: 


$$
\left(\begin{array}{rrr}
\langle 1, d, J+1, M|H| 1, d, J+1, M\rangle & \langle 1, d, J+1, M|H| 1, c, J, M\rangle & \langle 1, d, J+1, M|H| 2, c, J, M\rangle \\
\langle 1, c, J, M|H| 1, d, J+1, M\rangle & \langle 1, c, J, M|H| 1, c, J, M\rangle & \langle 1, c, J, M|H| 2, c, J, M\rangle \\
\langle 2, c, J, M|H| 1, d, J+1, M\rangle & \langle 2, c, J, M|H| 1, c, J, M\rangle & \langle 2, c, J, M|H| 2, c, J, M\rangle
\end{array}\right) .
$$

Or for a quasi-degeneracy between the $|1, d, J, M\rangle$ and $|3, d, J, M\rangle$ states of negative parity, the $3 \times 3$ Hamiltonian matrix will also have matrix elements due to the $|1, c, J-1, M\rangle$ state:

$$
\left(\begin{array}{rrr}
\langle 1, c, J-1, M|H| 1, c, J-1, M\rangle & \langle 1, c, J-1, M|H| 1, d, J, M\rangle & \langle 1, c, J-1, M|H| 3, d, J, M\rangle \\
\langle 1, d, J, M|H| 1, c, J-1, M\rangle & \langle 1, d, J, M|H| 1, d, J, M\rangle & \langle 1, d, J, M|H| 3, d, J, M\rangle \\
\langle 3, d, J, M|H| 1, c, J-1, M\rangle & \langle 3, d, J, M|H| 1, d, J, M\rangle & \langle 3, d, J, M|H| 3, d, J, M\rangle
\end{array}\right) .
$$

The mixed- $J$ matrix elements occur only for the Zeeman part of the Hamiltonian and can be derived from Eqs. (A23)(A30) and (A31)-(A36) of the Appendix. For the above examples these are:

$$
\begin{aligned}
\langle 1, d, J+1, M|H| 1, c, J, M\rangle= & \left\langle 1, d, J+1, M\left|H_{Z}\right| 1, c, J, M\right\rangle \\
= & g_{S} \frac{\left[\left(J+\frac{1}{2}\right)\left(J+\frac{3}{2}\right)(J+M+1)(J-M+1)^{1 / 2}\right.}{(J+1)[(2 J+1)(2 J+3)]^{1 / 2}} \mu_{B} \mathcal{H}, \\
\langle 1, d, J+1, M|H| 2, c, J, M\rangle= & 2^{-1 / 2} b L_{\Pi, \Sigma} \\
& \times \frac{\left[\left(J+\frac{1}{2}\right)\left(J+\frac{3}{2}\right)(J+M+1)(J-M+1)\right]^{1 / 2}}{(J+1)[(2 J+1)(2 J+3)]^{1 / 2}} \mu_{B} \mathcal{H} \\
& +2^{-1 / 2} a L_{\Pi, \Sigma} \\
& \times \frac{\left[\left(J+\frac{3}{2}\right)\left(J-\frac{1}{2}\right)(J+M+1)(J-M+1)\right]^{1 / 2}}{(J+1)[(2 J+1)(2 J+3)]^{1 / 2}} \mu_{B} \mathcal{H}, \\
\langle 1, c, J-1, M|H| 1, d, J, M\rangle= & g_{S} \frac{\left[\left(J+\frac{1}{2}\right)\left(J-\frac{1}{2}\right)(J+M)(J-M)\right]^{1 / 2}}{J[(2 J+1)(2 J-1)]^{1 / 2}} \mu_{B} \mathcal{H}, \\
\langle 1, c, J-1, M|H| 3, d, J, M\rangle= & 2^{-1 / 2} L_{\Pi, \Sigma} a \frac{\left[\left(J+\frac{1}{2}\right)\left(J-\frac{1}{2}\right)(J+M)(J-M)\right]^{1 / 2}}{J[(2 J+1)(2 J-1)]^{1 / 2}} \mu_{B} \mathcal{H} \\
& +2^{-1 / 2} L_{\Pi, \Sigma} b \frac{\left[\left(J+\frac{3}{2}\right)\left(J+\frac{1}{2}\right)(J+M)(J-M)\right]^{1 / 2}}{J[(2 J+1)(2 J-1)]^{1 / 2}} \mu_{B} \mathcal{H} .
\end{aligned}
$$

In the present case of the $\mathrm{CaH}$ molecule, the $N^{\prime}=1$ states with $J^{\prime}=3 / 2$ and $1 / 2$ from within the $B^{2} \Sigma, v^{\prime}=0$ state manifold are perturbed by the close-lying $J^{\prime}=3 / 2$ state from within the $A^{2} \Pi_{3 / 2}, v^{\prime}=1$ manifold (whose spin-orbit coupling constant, $A>0$ ). Hence the interacting states have negative parity and the relevant $3 \times 3$ Hamiltonian matrix takes the form of Eq. (27).

\section{COMPARISON OF THEORY AND EXPERIMENT}

Figure 4 shows the measured (points) spectral shifts (as derived from the positions of the maxima of the distribution curves of Fig. 2) as a function of the magnetic field strength at the edge of the trap, $\mathcal{H}_{\text {edge }}$. The shifts are linear in field strength and correspond to a small difference $\left(0.02 \mu_{B}\right)$ in the magnetic moments between the ground and excited states. Taking the field-free parameters of $\mathrm{CaH}$ from Berg and Klyning ${ }^{6}$ and Martin $^{7}$ (see caption) and approximating
$L_{\Pi, \Sigma} \equiv\left\langle\Pi\left|\mathbf{L}^{+}\right| \Sigma\right\rangle \quad$ by $2\left\langle\Pi\left|\mathbf{B L}_{y}\right| \Sigma\right\rangle / B_{\Sigma} \equiv 2 Q / B_{\Sigma} \quad$ we obtained Zeeman curves for the $N^{\prime}=1, J^{\prime}=3 / 2, M^{\prime}= \pm 3 / 2$ that, after subtraction of the respective $N^{\prime \prime}=0, J^{\prime^{\prime}}=1 / 2, M^{\prime}$ $= \pm 1 / 2$ curves, yield a semiquantitative agreement with the observed Zeeman shifts (dashed lines). The positive Zeeman shift corresponds to the transition between the $N^{\prime \prime}=0, J^{\prime \prime}$ $=1 / 2, M^{\prime \prime}=1 / 2$ and the $N^{\prime}=1, J^{\prime}=3 / 2, M^{\prime}=3 / 2$ state, and the negative shift to the transition between the $N^{\prime \prime}=0, J^{\prime \prime}$ $=1 / 2, M^{\prime \prime}=-1 / 2$ and the $N^{\prime}=1, J^{\prime}=3 / 2, M^{\prime}=-3 / 2$ state. By fitting the calculated Zeeman shifts to the observed ones (full lines) we obtain $L_{\Pi, \Sigma}=0.4$. This value is about twice that of $2 Q / B_{\Sigma}$, i.e., quite at odds with the "pure precession"' hypothesis, see, e.g., Refs. 14 and 17. The analysis of extensive field-free spectra of $\mathrm{CaH}$ (Refs. 6,7) led earlier to a similar conclusion.

The calculated slope of the negative Zeeman shift is slightly steeper than that of the positive shift; this is due to 


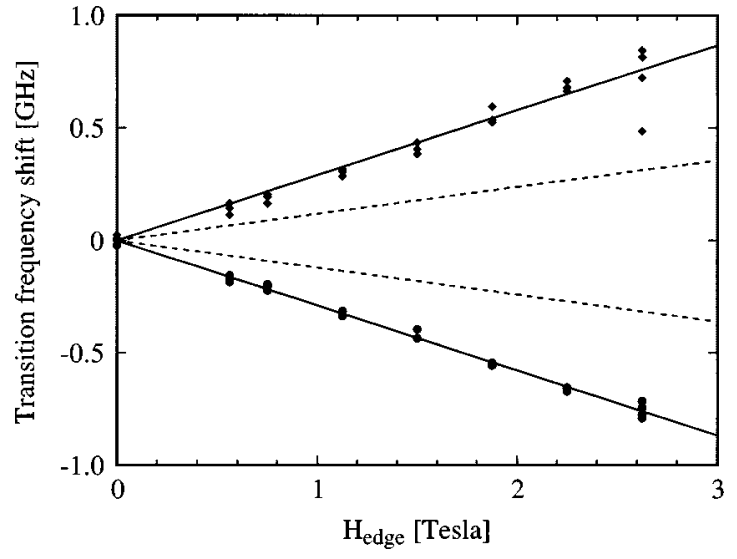

FIG. 4. Measured negative (circles) and positive (diamonds) spectral shifts as derived respectively from the type of data shown in Figs. $2 \mathrm{a}$ and $2 \mathrm{~b}$ as a function of the magnetic field strength at the edge of the trap, $\mathcal{H}_{\text {edge }}$. The dashed lines show a simulation based on a "pure precession" estimate of the $\left\langle\Pi\left|\mathbf{L}^{+}\right| \Sigma\right\rangle$ coupling between the $B, v^{\prime}=0$ and $A, v^{\prime}=1$ states, the full line is a fit to the experimental data yielding $\left\langle\Pi\left|\mathbf{L}^{+}\right| \Sigma\right\rangle=0.4$, see text. The values of the parameters that enter the calculation were taken from Berg and Klyning (Ref. 6) and from Martin (Ref. 7). These were: $B_{\Sigma}$ $=4.3455 \mathrm{~cm}^{-1}, \quad B_{\Pi}=4.2457 \mathrm{~cm}^{-1}, A=78.99 \mathrm{~cm}^{-1}, \Delta E=27.62 \mathrm{~cm}^{-1}$, $P=0.3, Q=0.333$.

the enhanced repulsion of the $N^{\prime}=1, J^{\prime}=3 / 2, M^{\prime}=3 / 2$ state that lies closer to the perturbing $A$-state.

We note that a field-induced hybridization of $\mathbf{J}$ may lead to the loss of a definite parity of the states, thus weakening the parity selection rule for the perturbation. ${ }^{15}$ We neglected this effect in our calculations.

\section{CONCLUSIONS}

We have observed splitting of the $B^{2} \Sigma, v^{\prime}=0\left(N^{\prime}=1\right.$, $\left.J^{\prime}=3 / 2\right) \leftarrow X^{2} \Sigma, v^{\prime \prime}=0\left(N^{\prime \prime}=0, J^{\prime \prime}=1 / 2\right)$ transition of $\mathrm{CaH}$ in a magnetic trapping field. Theory to explain the observed splitting has been developed. A good agreement between the theory and our experiment has been found. We conclude that the splitting is due to the coupling of the $N^{\prime}=1$ states from within the $B^{2} \Sigma, v^{\prime}=0$ state manifold with the close-lying $J^{\prime}=3 / 2$ state from within the $A^{2} \Pi_{3 / 2}, v^{\prime}=1$ manifold. The mixing of the $B$ and $A$-states results in a modification of the $B$-state magnetic dipole moment (and other properties). Fitting of the experimental magnetic shifts yields a value $L_{\Pi, \Sigma}=0.4$ of the interaction parameter that describes the coupling between the $B$ and $A$ states. This is about twice the value predicted by the "pure precession" hypothesis. ${ }^{17}$

\section{ACKNOWLEDGMENTS}

We thank R.W. Field (MIT) for enlightening discussions on rotational perturbations and the Zeeman effect in $\mathrm{CaH}$. This material is based upon work supported by the National Science Foundation under Grant No. PHY-9511951. J.D.W. is supported by a National Science Foundation Graduate Research Fellowship.

\section{APPENDIX}

\section{The field-free Hamiltonian and its matrix elements in Hund's case (a) basis}

The matrix elements of the field-free Hamiltonian of a linear molecule in the Hund's case (a) basis that couple the ${ }^{2} \Sigma$ and ${ }^{2} \Pi$ states in question, first given by van Vleck, ${ }^{16}$ are reproduced here for convenience:

$$
\begin{aligned}
& \left\langle{ }^{2} \Sigma_{1 / 2} J\left|H_{0}\right|^{2} \Sigma_{1 / 2} J\right\rangle=\left\langle{ }^{2} \Sigma_{-1 / 2} J\left|H_{0}\right|^{2} \Sigma_{-1 / 2} J\right\rangle \\
& =B_{\Sigma}\left[J(J+1)+\frac{1}{4}\right]+\Delta E \equiv \alpha, \\
& \left\langle{ }^{2} \Pi_{1 / 2} J\left|H_{0}\right|^{2} \Pi_{1 / 2} J\right\rangle=\left\langle{ }^{2} \Pi_{-1 / 2} J\left|H_{0}\right|^{2} \Pi_{-1 / 2} J\right\rangle \\
& =B_{\Pi}\left(J(J+1)+\frac{1}{4}\right)-\frac{A}{2} \equiv \beta, \\
& \left\langle{ }^{2} \Pi_{3 / 2} J\left|H_{0}\right|^{2} \Pi_{3 / 2} J\right\rangle=\left\langle{ }^{2} \Pi_{-3 / 2} J\left|H_{0}\right|^{2} \Pi_{-3 / 2} J\right\rangle \\
& =B_{\Pi}\left(J(J+1)-\frac{7}{4}\right)+\frac{A}{2} \equiv \gamma, \\
& \left\langle{ }^{2} \Sigma_{1 / 2} J\left|H_{0}\right|^{2} \Sigma_{-1 / 2} J\right\rangle=\left\langle{ }^{2} \Sigma_{-1 / 2} J\left|H_{0}\right|^{2} \Sigma_{1 / 2} J\right\rangle \\
& =B_{\Sigma}\left(J+\frac{1}{2}\right) \equiv(-1)^{s} \delta, \\
& \left\langle{ }^{2} \Pi_{3 / 2} J\left|H_{0}\right|^{2} \Sigma_{1 / 2} J\right\rangle=\left\langle{ }^{2} \Pi_{-3 / 2} J\left|H_{0}\right|^{2} \Sigma_{-1 / 2} J\right\rangle \\
& =B_{\Pi}\left[\left(J-\frac{1}{2}\right)\left(J+\frac{3}{2}\right)\right]^{1 / 2} \equiv \varepsilon, \\
& \left\langle{ }^{2} \Pi_{1 / 2} J\left|H_{0}\right|^{2} \Sigma_{-1 / 2} J\right\rangle=(-1)^{s}\left\langle{ }^{2} \Pi_{-1 / 2} J\left|H_{0}\right|^{2} \Sigma_{1 / 2} J\right\rangle \\
& =2 Q\left(J+\frac{1}{2}\right) \equiv(-1)^{s} \xi, \\
& \left\langle{ }^{2} \Pi_{3 / 2} J\left|H_{0}\right|^{2} \Sigma_{1 / 2} J\right\rangle=(-1)^{s}\left\langle{ }^{2} \Pi_{-3 / 2} J\left|H_{0}\right|^{2} \Sigma_{-1 / 2} J\right\rangle \\
& =2 Q\left[\left(J-\frac{1}{2}\right)\left(J+\frac{3}{2}\right)\right]^{1 / 2} \equiv \eta, \\
& \left\langle{ }^{2} \Sigma_{1 / 2} J\left|H_{0}\right|^{2} \Pi_{1 / 2} J\right\rangle=(-1)^{s}\left\langle{ }^{2} \Sigma_{-1 / 2} J\left|H_{0}\right|^{2} \Pi_{-1 / 2} J\right\rangle \\
& =P+2 Q \equiv \theta,
\end{aligned}
$$

with $\Delta E$ the energy difference between the zero-point energies of the $\Sigma$ and $\Pi$ states, $B_{\Sigma}$ and $B_{\Pi}$ their respective rotational constants, $A$ the spin-orbit splitting constant of the $\Pi$ state, and

$$
\begin{aligned}
& P \equiv\left\langle\Pi\left|\mathbf{A} \mathbf{L}_{y}\right| \Sigma\right\rangle=\frac{1}{2}\left\langle\Pi\left|\mathbf{A} \mathbf{L}^{+}\right| \Sigma\right\rangle, \\
& Q \equiv\left\langle\Pi\left|\mathbf{B} \mathbf{L}_{y}\right| \Sigma\right\rangle=\frac{1}{2}\left\langle\Pi\left|\mathbf{B} \mathbf{L}^{+}\right| \Sigma\right\rangle,
\end{aligned}
$$

the rotational perturbation parameters; $s=0$ or 1 depending on whether the $\Lambda=0$ state is $\Sigma^{+}$or $\Sigma^{-}$.

\section{The Zeeman Hamiltonian and its matrix elements in Hund's case (a) basis}

The Zeeman Hamiltonian is given by

$$
H_{Z}=-\mu_{Z} \mathcal{H}
$$

with $\mu_{Z}$ the component of the molecular magnetic dipole moment operator along the space-fixed $Z$-axis whose direction is determined by $\mathcal{H}$. The magnetic dipole moment operator 


$$
\mu_{Z}=\frac{1}{2}\left(\boldsymbol{\Phi}_{Z}^{+} \mu^{-}+\boldsymbol{\Phi}_{Z}^{-} \mu^{+}\right)+\boldsymbol{\Phi}_{Z}^{z} \mu_{z}
$$

depends on the body-fixed magnetic dipole moment operators

$$
\begin{aligned}
& \mu^{ \pm}=-\left(g_{L} \mathbf{L}^{ \pm}+g_{S} \mathbf{S}^{ \pm}\right) \mu_{B}, \\
& \mu_{z}=-\left(g_{L} \mathbf{L}_{z}+g_{S} \mathbf{S}_{z}\right) \mu_{B}
\end{aligned}
$$

(with $g_{L}=1$ and $g_{S} \simeq 2.0023$ the orbital and spin gyromagnetic ratios and $\mu_{B}$ the Bohr magneton) and the direction cosine operator $\boldsymbol{\Phi}$ with components

$$
\Phi_{F}^{g}=\mathbf{F} \cdot \mathbf{g},
$$

where $\mathbf{F} \equiv \mathbf{X}, \mathbf{Y}, \mathbf{Z}$ and $\mathbf{g} \equiv \mathbf{x}, \mathbf{y}, \mathbf{Z}$ are unit vectors defining righthanded Cartesian space- and body-fixed coordinate systems. As a result,

$$
\begin{aligned}
H_{Z}= & {\left[\frac{1}{2}\left(\boldsymbol{\Phi}_{Z}^{+} \mathbf{L}^{-}+\boldsymbol{\Phi}_{Z}^{-} \mathbf{L}^{+}\right)+\boldsymbol{\Phi}_{Z}^{z} \mathbf{L}_{z}\right] \mu_{B} \mathcal{H} } \\
& +\left[\frac{1}{2}\left(\boldsymbol{\Phi}_{Z}^{+} \mathbf{S}^{-}+\boldsymbol{\Phi}_{Z}^{-} \mathbf{S}^{+}\right)+\boldsymbol{\Phi}_{Z}^{z} \mathbf{S}_{z}\right] g_{S} \mu_{B} \mathcal{H} \\
= & \left(\mathbf{L}_{Z}+g_{S} \mathbf{S}_{Z}\right) \mu_{B} \mathcal{H} .
\end{aligned}
$$

The Hund's case (a) matrix elements of $\mathbf{L}$ and $\mathbf{S}$ in the bodyfixed, nonrotating frame are ${ }^{17,18}$

(1) $\Delta \Lambda= \pm 1, \Delta \Sigma=0$ :

$$
\left\langle L \Lambda \pm 1 S \Sigma\left|\mathbf{L}^{ \pm}\right| L \Lambda S \Sigma\right\rangle=\left\langle\Lambda \pm 1\left|\mathbf{L}^{ \pm}\right| \Lambda\right\rangle \equiv L_{\Lambda \pm 1, \Lambda}
$$

(2) $\Delta \Lambda=0, \Delta \Sigma= \pm 1$ :

$$
\left\langle L \Lambda S \Sigma \pm 1\left|\mathbf{S}^{ \pm}\right| L \Lambda S \Sigma\right\rangle=[S(S+1)-\Sigma(\Sigma \pm 1)]^{1 / 2} \text {; }
$$

(3) $\Delta \Lambda=\Delta \Sigma=0$ :

$$
\left\langle L \Lambda S \Sigma\left|\mathbf{L}_{z}\right| L \Lambda S \Sigma\right\rangle=\Lambda \text {; }
$$

(4) $\Delta \Lambda=\Delta \Sigma=0$ :

$$
\left\langle L \Lambda S \Sigma\left|\mathbf{S}_{z}\right| L \Lambda S \Sigma\right\rangle=\Sigma .
$$

As a result, for the states of interest:

$$
\begin{aligned}
\left\langle{ }^{2} \Sigma_{ \pm 1 / 2}\left|\mathbf{S}_{z}\right|^{2} \Sigma_{ \pm 1 / 2}\right\rangle & = \pm \frac{1}{2}, \\
\left\langle{ }^{2} \Sigma_{ \pm 1 / 2}\left|\mathbf{S}^{ \pm}\right|{ }^{2} \Sigma_{\mp 1 / 2}\right\rangle & =\left\langle{ }^{2} \Pi_{1 / 2}\left|\mathbf{S}^{-}\right|{ }^{2} \Pi_{3 / 2}\right\rangle \\
& =\left\langle{ }^{2} \Pi_{3 / 2}\left|\mathbf{S}^{+}\right|{ }^{2} \Pi_{1 / 2}\right\rangle \\
& =\left\langle{ }^{2} \Pi_{-1 / 2}\left|\mathbf{S}^{+}\right|^{2} \Pi_{-3 / 2}\right\rangle \\
& =\left\langle{ }^{2} \Pi_{-3 / 2}\left|\mathbf{S}^{-}\right|^{2} \Pi_{-1 / 2}\right\rangle=1, \\
\left\langle\Pi_{ \pm 1 / 2}\left|\mathbf{S}_{z}\right|^{2} \Pi_{ \pm 1 / 2}\right\rangle & =\mp \frac{1}{2}, \\
\left\langle\left.\Pi_{ \pm 3 / 2}|| \mathbf{S}_{z}\right|^{2} \Pi_{ \pm 3 / 2}\right\rangle & = \pm \frac{1}{2}, \\
\left\langle\Pi_{ \pm 1 / 2}\left|\mathbf{L}_{z}\right|^{2} \Pi_{ \pm 1 / 2}\right\rangle & =\left\langle\left.{ }^{2} \Pi_{ \pm 3 / 2}|| \mathbf{L}_{z}\right|^{2} \Pi_{ \pm 3 / 2}\right\rangle= \pm 1, \\
\left\langle{ }^{2} \Sigma_{ \pm 1 / 2}\left|\mathbf{L}^{ \pm}\right|^{2} \Pi_{\mp 1 / 2}\right\rangle & =\left\langle{ }^{2} \Sigma_{1 / 2}\left|\mathbf{L}^{-}\right|{ }^{2} \Pi_{3 / 2}\right\rangle \\
& =\left\langle\Pi_{3 / 2}\left|\mathbf{L}^{+}\right|{ }^{2} \Sigma_{1 / 2}\right\rangle \\
& =\left\langle{ }^{2} \Sigma_{-1 / 2}\left|\mathbf{L}^{+}\right|{ }^{2} \Pi_{-3 / 2}\right\rangle \\
& =\left\langle\Pi_{-3 / 2}\left|\mathbf{L}^{-}\right|{ }^{2} \Sigma_{-1 / 2}\right\rangle=L_{\Pi, \Sigma} .
\end{aligned}
$$

The direction cosine matrix elements along the space-fixed $Z$-axis can be obtained from Hougen's table ${ }^{19}$
(1) $\Delta \Omega= \pm 1, \Delta J=\Delta M=0$ :

$$
\left\langle\Omega \pm 1 J M\left|\mathbf{\Phi}_{Z}^{\mp}\right| \Omega J M\right\rangle=\frac{[(J \mp \Omega)(J \pm \Omega+1)]^{1 / 2}}{J(J+1)} M ;
$$

(2) $\Delta \Omega= \pm 1, \Delta J=+1, \Delta M=0$ :

$$
\begin{aligned}
\langle\Omega & \left. \pm 1 J+1 M\left|\boldsymbol{\Phi}_{Z}^{\mp}\right| \Omega J M\right\rangle \\
=\mp & \frac{[(J \pm \Omega+1)(J \pm \Omega+2)(J+M+1)(J-M+1)]^{1 / 2}}{(J+1)[(2 J+1)(2 J+3)]^{1 / 2}} ;
\end{aligned}
$$

(3) $\Delta \Omega= \pm 1, \Delta J=-1, \Delta M=0$

$$
\begin{aligned}
\langle\Omega & \left. \pm 1 J-1 M\left|\mathbf{\Phi}_{Z}^{\mp}\right| \Omega J M\right\rangle \\
= & \pm \frac{[(J \mp \Omega)(J \mp \Omega-1)(J+M)(J-M)]^{1 / 2}}{J[(2 J+1)(2 J-1)]^{1 / 2}} ;
\end{aligned}
$$

(4) $\Delta \Omega=\Delta J=\Delta M=0$

$$
\left\langle\Omega J M\left|\boldsymbol{\Phi}_{Z}^{z}\right| \Omega J M\right\rangle=\frac{\Omega M}{J(J+1)} ;
$$

(5) $\Delta \Omega=0, \Delta J=+1, \Delta M=0$

$$
\begin{aligned}
\left\langle\Omega J+1 M\left|\boldsymbol{\Phi}_{Z}^{z}\right| \Omega J M\right\rangle & \\
= & \frac{[(J+\Omega+1)(J-\Omega+1)(J+M+1)(J-M+1)]^{1 / 2}}{(J+1)[(2 J+1)(2 J+3)]^{1 / 2}} ;
\end{aligned}
$$

(6) $\Delta \Omega=0, \Delta J=-1, \Delta M=0$

$$
\begin{aligned}
\left\langle\Omega J-1 M\left|\boldsymbol{\Phi}_{Z}^{z}\right| \Omega J M\right\rangle & \\
= & \frac{[(J+\Omega)(J-\Omega)(J+M)(J-M)]^{1 / 2}}{J[(2 J+1)(2 J-1)]^{1 / 2}} .
\end{aligned}
$$

Hence, for instance

$$
\begin{aligned}
& \left\langle{ }^{2} \Sigma_{1 / 2}, J-1, M\left|H_{Z}\right|^{2} \Pi_{3 / 2}, J, M\right\rangle \\
& =-L_{\Pi, \Sigma} \frac{\left[\left(J+\frac{3}{2}\right)\left(J+\frac{1}{2}\right)(J+M)(J-M)\right]^{1 / 2}}{2 J[(2 J+1)(2 J-1)]^{1 / 2}} \mu_{B} \mathcal{H} .
\end{aligned}
$$

${ }^{1}$ B. Friedrich, R. deCarvalho, J. Kim, D. Patterson, J. D. Weinstein, and J. M. Doyle, J. Chem. Soc., Faraday Trans. 94, 1783 (1998).

${ }^{2}$ J. Weinstein, R. deCarvalho, T. Guillet, B. Friedrich, and J. M. Doyle, Nature (London) 395, 148 (1998).

${ }^{3}$ B. Friedrich and D. R. Herschbach, Phys. Rev. Lett. 74, 4623 (1995).

${ }^{4}$ T. Takekoshi, B. M. Patterson, and R. J. Knize, Phys. Rev. Lett. 81, 5105 (1998).

${ }^{5}$ J. M. Doyle, B. Friedrich, J. Kim, and D. Patterson, Phys. Rev. A 52, R2515 (1995).

${ }^{6}$ L. E. Berg and L. Klyning, Phys. Scr. 10, 331 (1974).

${ }^{7}$ H. Martin, J. Mol. Spectrosc. 108, 66 (1984).

${ }^{8}$ J. Kim, B. Friedrich, D. P. Katz, D. Patterson, R. deCarvalho, J. D. Weinstein, and J. M. Doyle, Phys. Rev. Lett. 78, 3665 (1997).

${ }^{9}$ J. D. Weinstein, R. deCarvalho, J. Kim, D. Patterson, B. Friedrich, and J. M. Doyle, Phys. Rev. A 57, R3173 (1998).

${ }^{10}$ T. Leininger and G. Jeung, J. Chem. Phys. 103, 3942 (1995).

${ }^{11}$ L. E. Berg and L. Klyning, Astron. Astrophys., Suppl. Ser. 13, 325 (1974).

${ }^{12}$ G. Herzberg, Spectra of Diatomic Molecules (Van Nostrand, Princeton, 1950).

${ }^{13}$ H. E. Radford and H. P. Broida, Phys. Rev. 128, 231 (1962).

${ }^{14}$ H. E. Radford, Phys. Rev. 126, 1035 (1962). 
${ }^{15}$ B. Friedrich, Int. Rev. Phys. Chem. 14, 113 (1995).

${ }^{16}$ J. H. Van Vleck, Phys. Rev. 33, 467 (1929).

${ }^{17}$ H. Lefebvre-Brion and R. W. Field, Perturbations in the Spectra of Diatomic Molecules (Academic, New York, 1986).
${ }^{18}$ R. N. Zare, Angular Momentum (Wiley, New York, 1988).

${ }^{19} \mathrm{~J}$. T Hougen, The calculations of rotational energy levels and rotational line intensities in diatomic molecules, National Bureau of Standards Monograph 115, Washington, D.C., 1970. 